\title{
Light emission from silicon nanocrystals: Probing a single quantum dot
}

\author{
I. Sychugov ${ }^{\text {a }}$, R. Juhasz ${ }^{\text {a }}$ J. Valenta ${ }^{\mathrm{b}}$, M. Zhang ${ }^{\mathrm{c}}$, P. Pirouz $^{\mathrm{c}}$, J. Linnros $^{\mathrm{a}, *}$ \\ ${ }^{a}$ Laboratory of Materials and Semiconductor Physics, Royal Institute of Technology, SE-16440 Kista-Stockholm, Sweden \\ ${ }^{\mathrm{b}}$ Faculty of Mathematics and Physics, Department of Chemical Physics and Optics, Charles University, \\ Ke Karlovu 3, Prague 2, CZ-12116, Czech Republic \\ ${ }^{\mathrm{c}}$ Department of Materials Science and Engineering, Case Western Reserve University, Cleveland, OH 44106-7204, USA
}

Available online 18 January 2006

\begin{abstract}
Analysis of low-temperature photoluminescence measurements performed on single silicon nanocrystals is presented. The luminescence emission linewidth of Si nanocrystals is found to be less than thermal broadening at low temperature, confirming the atomic-like nature of their energetic states. Beside the main peak the low-temperature spectra reveal a $\sim 6 \mathrm{meV}$ replica, the origin of which is discussed. For some of the investigated dots, we also observe a $\sim 60 \mathrm{meV}$ transverse optical (TO) phonon replica. The regular arrangement of individual nanocrystals used in this work enables combined high-resolution transmission electron microscopy (TEM) and low-temperature photoluminescence characterization of the same single quantum dot.
\end{abstract}

(C) 2005 Elsevier B.V. All rights reserved.

PACS: 78.67.Hc; 78.55.Ap

Keywords: Silicon nanocrystal; Luminescence

\section{Introduction}

Semiconductor nanocrystals exhibit a number of properties, which differ from characteristics of corresponding bulk materials. First, as a result of the quantum confinement effect, the bandgap energy increases when nanocrystal size approaches the Bohr exciton radius. The embedding of nanocrystals into a media with higher bandgap energy, on the other hand, prevents carriers from migration out of the nanocrystals, thus promoting radiative electron-hole recombination. In addition, discrete energy levels of a quantum dot lead to sharp emission lines for optical transitions between excited and ground states. Therefore, such nanostructures are often referred to as "artificial atoms".

The luminescent properties of silicon nanocrystals were first explored in porous $\mathrm{Si}$ [1] and were later studied in $\mathrm{Si}$ nanocrystals formed by various techniques, mostly resulting in nanocrystals passivated by silicon dioxide [2]. In such a case, using an analogy with well-explored direct bandgap quantum dots, one may say that Si nanocrystals have a "wetting layer"

\footnotetext{
* Corresponding author. Tel.: +46 8 7904370; fax: +46 87527782 .

E-mail address: linnros@imit.kth.se (J. Linnros).
}

or are over-coated with a material of a high bandgap value of about $9 \mathrm{eV}$. This means that carrier injection into such nanocrystals is hindered and special measures should be taken in order to electrically excite luminescence from them [3].

Normally, the observed luminescence spectra from silicon nanocrystals are very broad, a few hundred millielectron volt even at low temperatures, which has generally been attributed to inhomogeneous line-broadening. In ordinary spectroscopic measurements an ensemble of quantum dots (with a broad distribution of energetic states) is addressed rather than single nanocrystals. Consequently, valuable information on its level structure, emission band and possible individual variations from dot to dot are blurred. However, the relatively large linewidth at room temperature found even for an individual nanocrystal [4] raised some doubts on the luminescence mechanism being that of a pure Si quantum dot.

Previously it was shown that the procedure of nanocrystal formation by electron beam lithography, plasma etching and subsequent size-reduction by a careful oxidation scheme may result in well-resolved arrays of single $\mathrm{Si}$ nanocrystals [4]. Recently, low temperature measurements demonstrated linewidths of individual nanocrystals less than the thermal broadening at this temperature, proving atomic-like emission from silicon quantum dots subject to quantum confinement 
[5]. Furthermore, a $\sim 6 \mathrm{meV}$ replica of the main peak was observed at low temperatures, as well as a $\sim 60 \mathrm{meV}$ replica, attributed to transverse optical (TO) phonons. Intriguingly, this TO replica is only present in a fraction of the analyzed dots. Here, we discuss these findings in terms of different physical scenarios and underline the importance of both structural and electronic configuration characterization of a single nanocrystal.

\section{Experimental}

Samples in the form of pillar arrays were fabricated using electron beam lithography and reactive ion etching. This was followed by oxidation to form nanocrystals at the pillar tops.

$N$-type $\mathrm{Si}$ wafers with a $25 \mathrm{~nm}$ thermal oxide were used as substrates and dots with a diameter of $\sim 100 \mathrm{~nm}$ were defined by an electron beam. Subsequent reactive ion etching produced $\sim 200 \mathrm{~nm}$ tall Si pillars (Fig. 1a). Thermal oxidation at $900{ }^{\circ} \mathrm{C}$ induced a non-uniform shrinking of the Si core of the pillars (Fig. 1b). A second oxidation led to the formation of a silicon nanocrystal at the top of pillars as a result of the self-
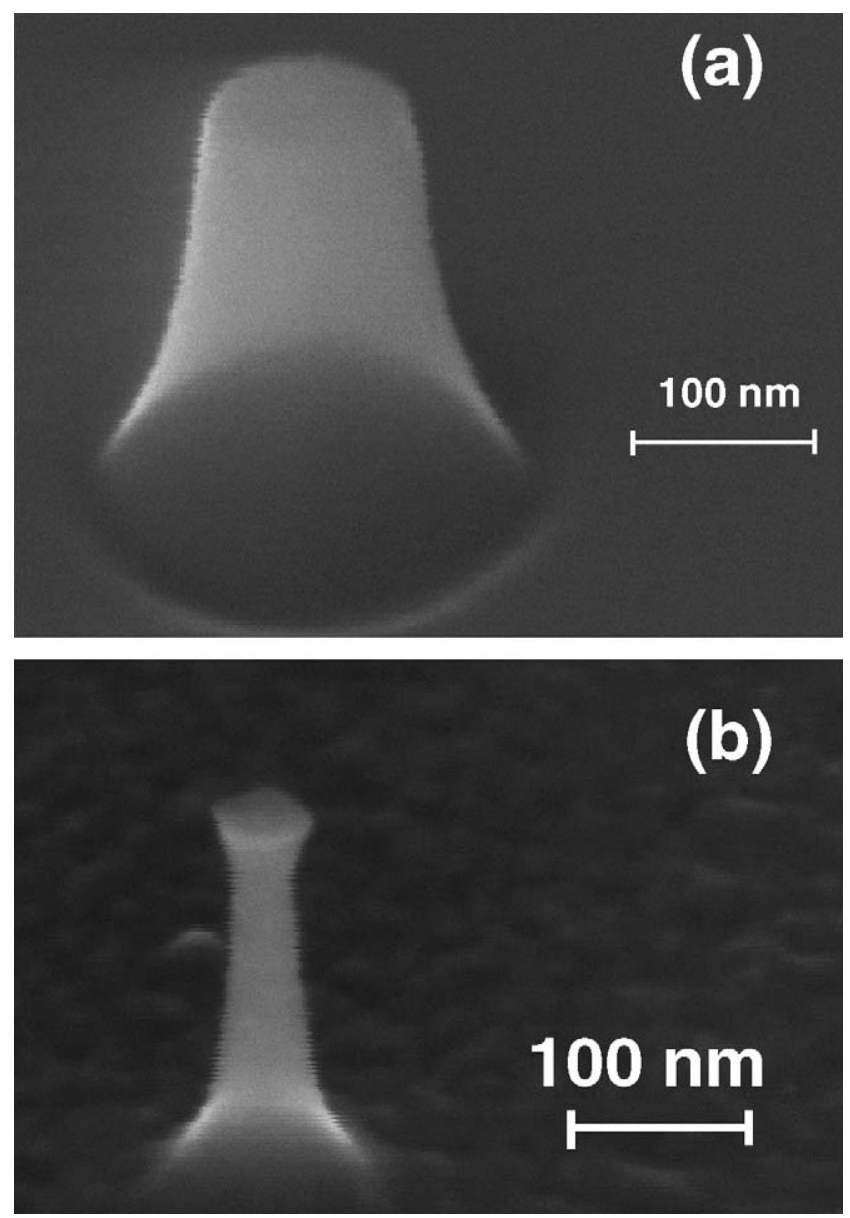

Fig. 1. SEM images (tilted $45^{\circ}$ ) of (a) a silicon pillar etched by reactive ionetching, using masks defined by e-beam lithography and (b) the same pillar after $5 \mathrm{~h}$ of oxidation at $900{ }^{\circ} \mathrm{C}$ and subsequent removal of $\mathrm{SiO}_{2}$. Non-uniform oxidation rate along pillar height results in the observed convex shape of sidewalls.
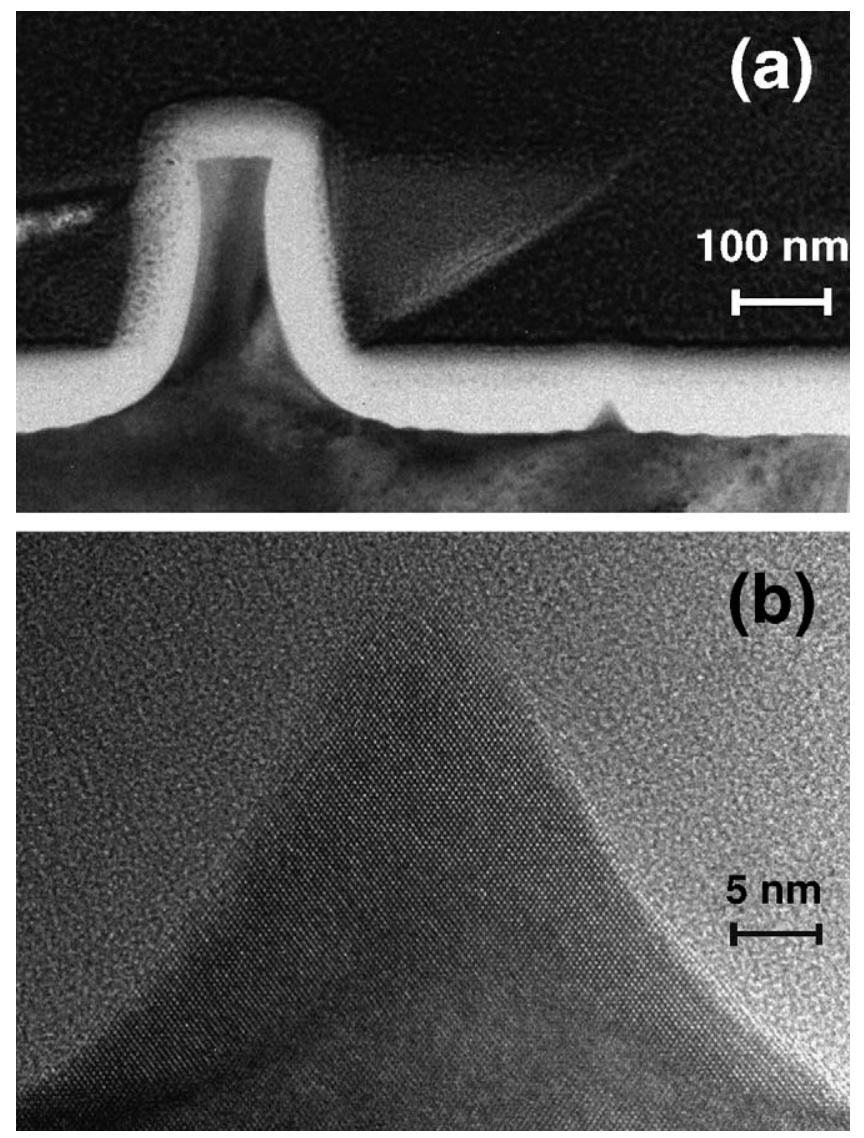

Fig. 2. (a) TEM micrograph of a silicon pillar after the first oxidation. The dark region inside the brighter shell corresponds to the silicon core embedded in a $\mathrm{SiO}_{2}$ matrix and (b) high-resolution TEM image of a smaller "satellite" pillar, seen in (a) to the right of the main pillar. The Si substrate used is $\left(\begin{array}{lll}0 & 0 & 1\end{array}\right)$ and the nanopillars have the same orientation as the substrate as illustrated by corresponding lattice fringes.

limiting oxidation, known to occur for a small radius of curvature. In Fig. 2a, transmission electron microscopy (TEM) images are shown for a pillar after the first oxidation step. Indeed, it is seen that the oxidation rate is faster at the "neck" region of the pillar, while at the top oxidation is partly suppressed due to the self-limiting effect. A small "satellite" pillar can also be seen in this figure and its high-resolution TEM (HRTEM) image is appended in Fig. 2b. Corresponding lattice fringes of crystalline $\mathrm{Si}$ are visible, which indicate that silicon pillars remain crystalline after high temperature treatment in the furnace. In order to prepare TEM specimens of pillars a focused ion beam (FIB) machine was used to cut out a foil, containing a row of pillars. In general, the feasibility of HRTEM characterization of such structures opens a way to probe both structural (from HRTEM) and electronic (from low-temperature photoluminescence) properties of the same single nanocrystal. However, additional experimental difficulties arise when, after the second oxidation step, a smaller pillar with a nanocrystal on top is addressed. No images of luminescent quantum dots have been obtained so far while further experiments are under way.

For PL characterization, samples were mounted on the cold finger of a liquid helium flow cryostat (Fig. 3). PL was excited 


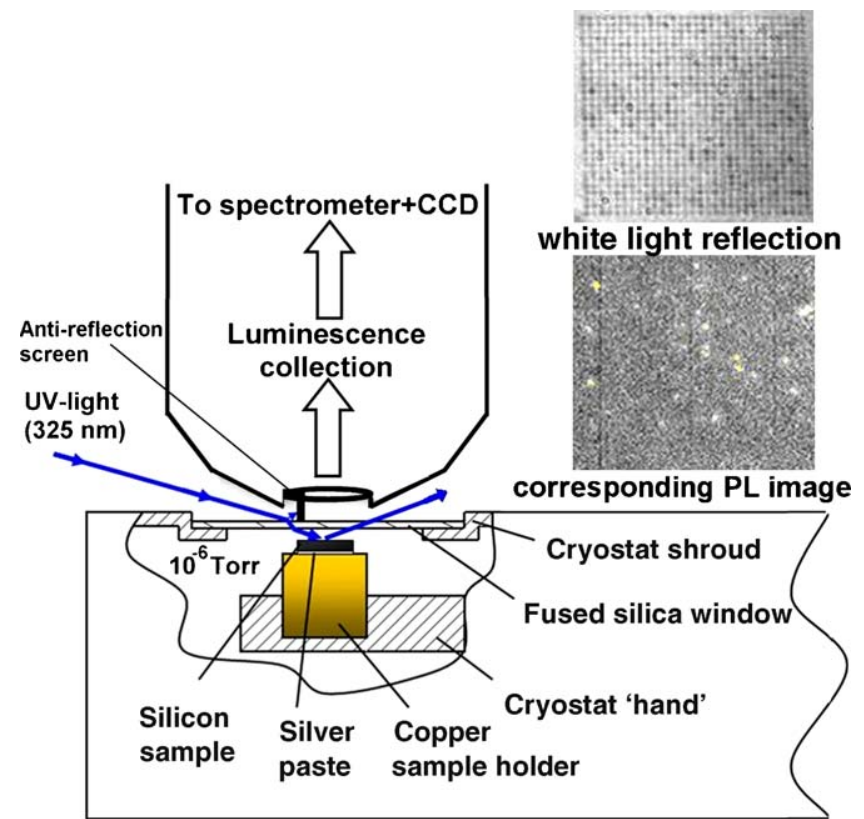

Fig. 3. Schematic representation of the PL setup. Luminescence is excited with UV-line $(325 \mathrm{~nm}$ ) of a He-Cd laser. A sample is mounted on a finger of a helium cooled cryostat. Emitted light is collected by a window-corrected objective with high numerical aperture $(\mathrm{NA}=0.7)$. Typical sample images are shown in the inset: a white light reflection image (top) and the corresponding PL image (bottom). Rows of silicon pillars can be discerned and each luminescence source from the PL image can be traced back to a certain pillar.

by the UV line $(325 \mathrm{~nm})$ of a cw He-Cd laser with pumping intensity $\sim 1 \mathrm{~W} / \mathrm{cm}^{2}$. The laser beam impinged on the sample surface at grazing incidence. To prevent reflected laser light from entering the objective, a tiny screen was used. The light emitted from a sample was collected by a window-corrected objective with a high numerical aperture $(\mathrm{NA}=0.7)$. An imaging spectrometer coupled to a liquid nitrogen cooled charge coupled device (CCD) camera was employed to detect the luminescence. Two different gratings of the imaging spectrometer provided spectral resolutions of 3 or $0.8 \mathrm{~nm}$. To get an acceptable signal to noise ratio each spectrum acquisition lasted at least for $30 \mathrm{~min}$. All spectra were corrected with the system response curve.

Inset in Fig. 3 is a white light reflected image of the pillar array with a pillar-to-pillar distance of $1 \mu \mathrm{m}$; the lower image shows the corresponding PL image under $325 \mathrm{~nm}$ excitation, revealing several well-separated light emitting objects. The periodic arrangement of pillars, provided by the e-beam, allows to establish exactly which pillar contains a luminescent nanocrystal. This particular pillar can be addressed by the TEM after PL characterization.

\section{Results and discussion}

When investigating temperature dependence of the luminescence line of a silicon nanocrystal we found that at decreasing temperature, there is a continuous line narrowing of the luminescence band and the occurrence of certain sidebands in some cases. At temperatures below $30 \mathrm{~K}$, however, we were

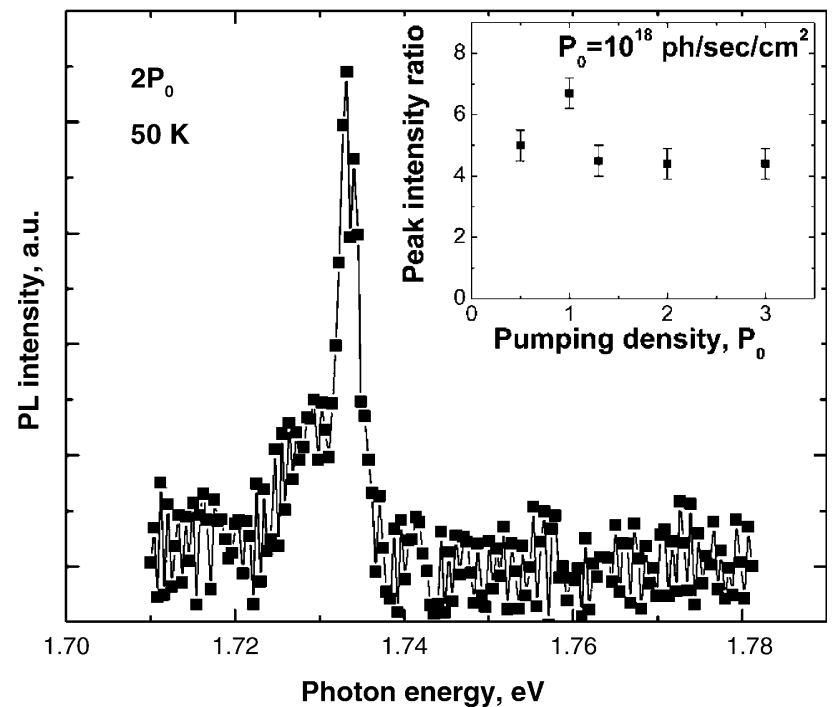

Fig. 4. Recorded spectrum from a silicon nanocrystal at $50 \mathrm{~K}$ under pumping photon flux $P_{0}=2 \times 10^{18}$ photons $/ \mathrm{s} / \mathrm{cm}^{2}$. Two peaks can be discerned: the main line and a somewhat broader satellite peak. We attribute the main peak to bandto-band recombination in a quantum-confined nanostructure, featuring a linewidth less than $k_{\mathrm{B}} T$ at this temperature $(\sim 5 \mathrm{meV})$. The lack of influence of the pumping intensity on the peak intensity ratio (see the inset) rules out possible biexciton nature of the replica. Tentatively, this satellite feature can be attributed to an acoustic phonon-assisted transition (see text).

unable to detect any consistent PL. We attribute this to the drastic lifetime increase observed for $\mathrm{Si}$ nanocrystals and porous $\mathrm{Si}$ at cryogenic temperatures [6]. This effect has previously been explained in terms of a singlet-triplet splitting with a lower lying 'dark' triplet state [6]. As the emission rate is lowered by a factor of about 50 , the emission rate seems to be below the detection capability of our measurement system. Above $30 \mathrm{~K}$, on the other hand, we were able to record singledot emission spectra and in Fig. 4, the PL spectrum at $50 \mathrm{~K}$ of a silicon nanocrystal is presented. It is seen that the emission line consists of a sharp peak (with a linewidth less than $k_{\mathrm{B}} T=5 \mathrm{meV}$ ) and a somewhat broader satellite, separated by $\sim 6 \mathrm{meV}$. The inset shows the ratio of these two peaks as a function of excitation power. Since there is no clear dependence, we rule out possible biexciton nature of the satellite peak. Instead this line might be attributed to a low frequency acoustic phonon assisted transition, taking into account torsional or spheroidal modes as calculated by Takagahara [7]. For a $4 \mathrm{~nm}$ diameter $\mathrm{Si}$ nanocrystal, he calculated a set of discrete values for the acoustic phonon energy spectrum starting from $\sim 5 \mathrm{meV}$. By Raman spectroscopy [8] the presence of confined acoustic modes with energy of a few millielectron volt was, indeed, experimentally observed.

When temperature increases to $80 \mathrm{~K}$, the fine structure of the peak can no longer be resolved and the main line and the replica are measured as a single peak. A summary of measured luminescence spectra parameters for various dots at this temperature is presented in Fig. 5. Note that a fraction of the dots $(\sim 30 \%)$ exhibit a silicon TO-phonon replica, standing $\sim 60 \mathrm{meV}$ apart from the main line with a position that does not 


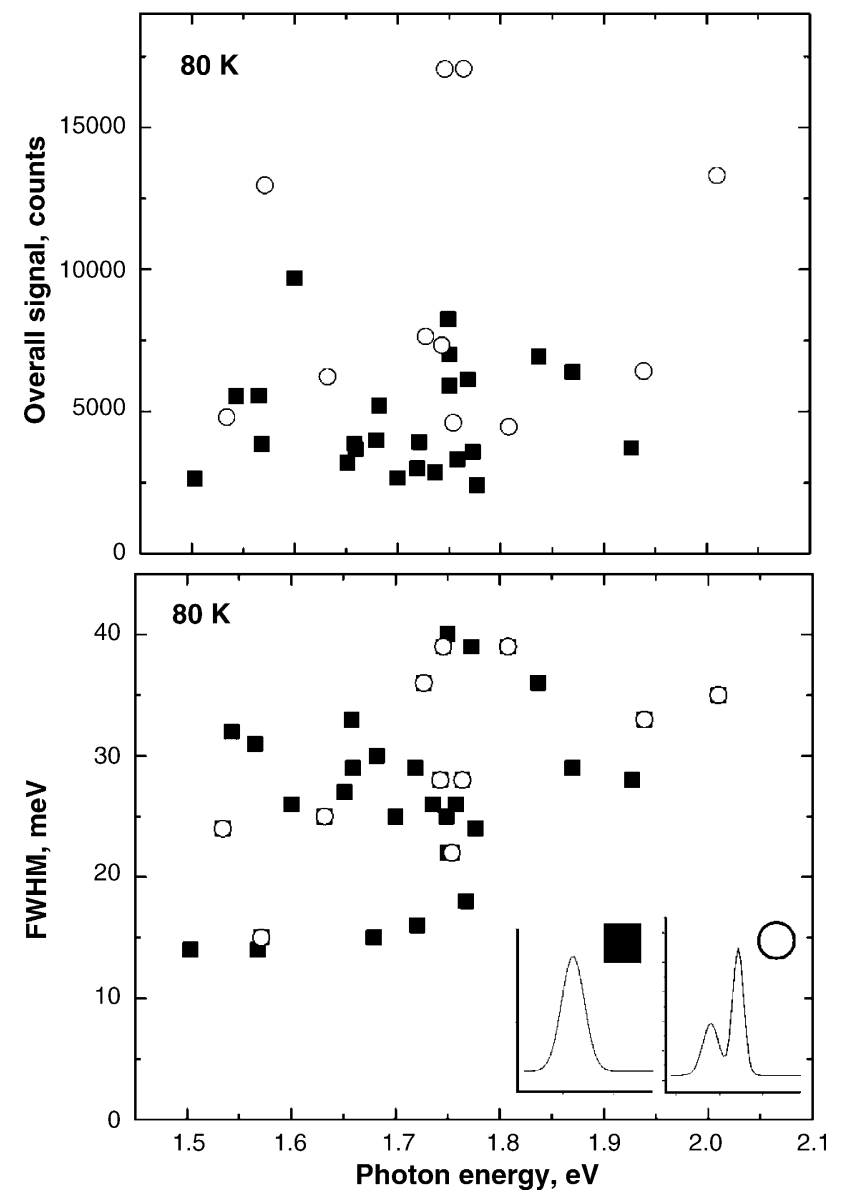

Fig. 5. Statistical summary of spectra for dots with various emission energies at $80 \mathrm{~K}$ : (a) signal intensity as a function of photon emission energy and (b) full width at half-maximum of the emission line as a function of its peak position. Open and dark circles correspond to the dots exhibiting and not exhibiting TOphonon replica at this temperature, respectively.

vary with peak emission energy (dot size). While this TO phonon involvement seems quite natural, it is not clear why only a fraction of the measured nanocrystals possess it. We may speculate that in some nanocrystals carriers are strongly localized (promoting no phonon PL) or that local differences in geometry may enhance or decrease the probability of the TO phonon-assisted process. In Fig. 5, dots exhibiting TO-phonon involvement are shown as open circles, while dark squares stand for nanocrystals without a TO-phonon assisted transition.

In Fig. 5a, the overall signal intensity is plotted versus the emission photon energy for a number of dots probed at $80 \mathrm{~K}$. It is seen that within the same spectra integration time (30 min.), the total light output varies significantly from dot-to-dot. We attribute such variations in quantum efficiency not only to possible differences in luminescence geometry, but also to the blinking phenomenon. This effect of sudden interruptions of light emission from a quantum dot has attracted much attention recently due to its complex physics and importance in nanocrystal applications. Indeed, blinking lowers the effective quantum efficiency for a quantum dot and thereby degrades the performance of any device built on ensembles of quantum dots. We found that characteristic switching times in silicon nanocrystals are in the order of seconds and vary from dotto-dot and with temperature [9]. It is commonly accepted, that blinking is governed by trap sites, surrounding a nanocrystal [10]. These sites are capable of capturing an excited carrier from the dot, thus making it charged and, consequently, dark. The dot comes back to its initial luminescent state when the carrier returns. However, the characteristic blinking time is found to be different for different nanocrystals. We ascribe such variations to the unique trap configurations for the surroundings of each dot.

In Fig. 5b, we present a statistical breakdown of the linewidth for different single silicon nanocrystals, measured at $80 \mathrm{~K}$. In general, the homogeneous width of a quantum dot is given by the inverse of the dephasing time, which consists of the radiative lifetime and various scattering times (interaction of the exciton with phonons, interface states, defects, etc.) [11]. Thus, the linewidth is a unique parameter of an individual quantum dot and depends on its interface with surrounding matrix, dot geometry and purity. Indeed, what we find is a scatter of this parameter without any distinct dependence on the dot size.

As shown above, many key luminescence parameters of a silicon nanocrystal vary significantly from one quantum dot to another. In order to get a better insight into the underlying physics of these variations, combined HRTEM and lowtemperature photoluminescence spectroscopy should be applied. Results of such characterization may also be found useful for theoretical modeling of nanocrystals and their electronic properties.

\section{Conclusions}

Silicon nanocrystals were studied by the micro photoluminescence technique in a range from liquid helium temperature to room temperature. It was shown that the emission linewidth of Si nanocrystals at low temperatures can be less than the thermal broadening, confirming the atomic-like nature of these low-dimensional entities. Also exciton-phonon interactions in Si nanocrystals were revealed as a TO phonon replica was observed and a $\sim 6 \mathrm{meV}$ replica, which we tentatively attribute to spheroidal or torsion acoustic modes. The observed dot-to-dot variation in luminescence properties highlights the need for both structural and electronic configuration characterization of a single nanocrystal.

\section{Acknowledgments}

The authors gratefully thank A. Galeckas for technical assistance. Financial support was received from the Swedish Research Council (VR). Participation of one of the authors (JV) was financed in the framework of Research Center LC510 by MSMT.

\section{References}

[1] L.T. Canham, Appl. Phys. Lett. 57 (1990) 1046; V. Lehmann, U. Gösele, Appl. Phys. Lett. 58 (1991) 856. 
[2] J. Linnros, N. Lalic, A. Galeckas, V. Grivickas, J. Appl. Phys. 86 (1999) 6128.

[3] R.G. Walters, H. Atwater, G. Bourianoff, Nat. Mater. 4 (2005) 143.

[4] J. Valenta, R. Juhasz, J. Linnros, Appl. Phys. Lett. 80 (2002) 1070.

[5] I. Sychugov, R. Juhasz, J. Valenta, J. Linnros, Phys. Rev. Lett. 94 (2005) 087405 .

[6] A.G. Cullis, L.T. Canham, P.D.J. Calcott, J. Appl. Phys. 82 (1997) 909.
[7] T. Takagahara, J. Lumin. 70 (1996) 129.

[8] M. Fujii, Y. Kanzawa, S. Hayashi, K. Yamamoto, Phys. Rev. B 54 (1996) R8373.

[9] I. Sychugov, R. Juhasz, J. Linnros, J. Valenta, Phys. Rev. B 71 (2005) 115331.

[10] A.L. Efros, M. Rosen, Phys. Rev. Lett. 78 (1997) 1110.

[11] T. Takagahara, Phys. Rev. Lett. 71 (1993) 3577. 